\title{
Effects of Kudingcha Nanoparticles in Hyperlipidaemic Rats Induced by a High Fat Diet
}

\author{
Hongliang Zhang ${ }^{a, b} \quad$ Xiaoqin Zou ${ }^{c} \quad$ Qiuyan Huang $^{d} \quad$ Xiaobin Zhong ${ }^{b}$ \\ Zhenguang Huanga
}

aThe department of pharmacy, The First Affiliated Hospital of Guangxi Medical University, Nanning, ${ }^{b}$ Guangxi Key Laboratory of Regenerative Medicine, The First Affiliated Hospital of Guangxi Medical University, Nanning, 'The department of Scientific Research, The First Affiliated Hospital of Guangxi Medical University, Nanning, dGuangxi institute of Chinese medicine pharmaceutical science, Nanning, China

\section{Key Words}

Kudingcha • Nanoparticles • Hyperlipidaemia

\begin{abstract}
Background/Aims: The herbal medicine Kudingcha has a bitter taste and low bioavailability for lipid reduction. To improve the bioavailability and ameliorate the compliance, we prepared Kudingcha nanoparticles and investigated their effect in hyperlipidaemic rats. In addition, the safety and lipid-lowering mechanism of the Kudingcha nanoparticles were examined. Methods: Kudingcha nanoparticles were prepared by ionotropic gelation and spray-drying. Seventy rats were randomly assigned into eight groups: a normal fat diet group (NF), a high-fat group (HF), a spontaneous recovery group (SR), a Kudingcha group (KDC), a blank nanoparticle group (B$\mathrm{N})$, and a Kudingcha nanoparticle groups (low, medium and high doses). All groups (except for the normal fat diet group) were fed a high-fat diet to establish hyperlipidaemia. Different interventions were administered to the treatment groups for four weeks. Serum lipids were measured using commercially available kits according to the recommended protocols. Liver morphology and histopathology were examined by a light microscope. The mRNA and protein levels of TLR4 and NF-KB were determined by RT-PCR and Western blotting, respectively. In addition, acute toxicity was evaluated by the $\mathrm{LD}_{50}$ test. Results: The Kudingcha nanoparticles were spherical and had a smooth surface. The size distribution of the nanoparticles was 100 $600 \mathrm{~nm}$. Acute toxicity results revealed that the Kudingcha nanoparticles were a non-toxic substance. Compared with regular Kudingcha, TG and TC decreased distinctly in the Kudingcha nanoparticles, especially for the moderate and high dose groups $(p<0.05)$. Moreover, the Kudingcha nanoparticles were superior in lowering body, liver and adipose tissue weights compared to Kudingcha $(p<0.05)$. With respect to antioxidant properties, the nanoparticles
\end{abstract}

$\mathrm{H}$. Zhang and X. Zou are co-first authors, and they contributed equally to this work.

Xiaobin Zhong and Zhenguang Huang
The First Affiliated Hospital of Guangxi Medical University

Shuangyong Road, Nanning, Guangxi (PR China)

Tel.+86-0771-5356154, E-Mail 1878125675@qq.com, 137109320@qq.com

\section{KARGER}




\section{Cellular Physiology Cell Physiol Biochem 2018;45:2257-2267

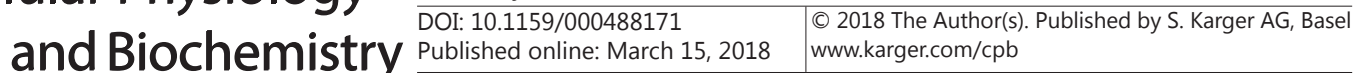 \\ Zhang et al.: Kudingcha Nanoparticles for the Treatment of Hyperlipidaemia}

also revealed an outstanding impact on serum SOD and MDA. In addition, liver morphology and histology in the moderate and high dose nanoparticle groups were similar to those in the normal group. Finally, the mRNA and protein expression levels of TLR4 and NF-KB in the liver tissue of the nanoparticle groups were significantly upregulated compared with the KDC group $(p<0.05)$. Conclusions: Based on these results, we conclude that Kudingcha nanoparticles are a potent lipid-lowering agent and may have a potential role in the treatment of hyperlipidaemia and fatty liver disease.

(C) 2018 The Author(s)

Published by S. Karger AG, Basel

\section{Introduction}

Hyperlipidaemia is an umbrella term that refers to any of several acquired or genetic disorders that result in a high level of lipids circulating in the blood. These lipids can enter the walls of arteries and increase the risk of developing atherosclerosis, which can lead to coronary heart disease, stroke and other types of cardiovascular disease [1]. Smoking, obesity, diabetes, hypertension, poor diet, and a sedentary lifestyle are also risk factors for atherosclerosis [2].

As mentioned in the literature reported by the Centers for Disease Control, hyperlipidaemia is second to hypertension in the list of the 10 most common chronic conditions [3]. Therefore, prevention and treatment of hyperlipidaemia have received considerable attention. Lifestyle modification, involving a healthy diet, regular exercise habits, no smoking, and maintenance of a healthy weight, remains the best strategy for reducing the risk of hyperlipidaemia. Additionally, medications known as statins and fibrates play an essential role in both the prevention and treatment of hyperlipidaemia.

Statins taken orally, usually once daily, are extremely effective in lowering LDL and VLDL levels. However, the most common side effects that lead individuals to stop taking statin medications are intolerable muscle and joint aches [4]. The degree of danger greatly rises when statins are used in combination with fibrates. In addition, statins may induce diabetes and cognitive dysfunction.

The use of alternative treatments, especially medicinal plants and their complements, to treat different diseases, such as hyperlipidaemia, diabetes, and cardiovascular disease, has rapidly increased worldwide over the recent decades [5-7]. Kudingcha (Broadleaf holly leaf CJ Tseng), a traditional Chinese herbal medicine, which is a common plant in Guangxi, a province of southwest of China, is one such alternative treatment. At present, Kudingcha is recorded in the Guangxi Zhuang Nationality Drug Quality Standards. Many studies have indicated that Kudingcha has the effect of reducing lipids [8]. However, due to the bitter taste and low bioavailability for lipid reduction, there are inevitable drawbacks of poor compliance and the need for large doses. Consequently, decreasing the dose and improving compliance is the goal that we are pursuing.

Over the past decades, nanotechnology has emerged as a promising research direction in the field of medicine [9]. Nanoparticles have remarkable properties, including a small particle size, a large surface area, passive targeting of the liver, etc. [10]. Nanoparticle vehicles address many of the challenges of drug delivery by improving drug stability, increasing bioavailability, and decreasing toxicity and side effects [11]. Therefore, we prepared nanoparticles of Kudingcha to solve the currently existing problems of renal damage and poor compliance associated with the free drug. In addition, nanoparticle-sized materials have markedly different physicochemical characteristics than their larger-sized versions; thus, they may have very different effects on living cells and can potentially result in toxicities even at low cumulative doses [12]. For this reason, the potential for adverse events and toxicities related to the nanoparticle vehicle itself must be considered, especially with novel nanoparticle formulations.

Although Kudingcha exhibits positive effects for hyperglycaemia and improves lipid metabolism, its definitive mechanism remains largely unknown. Toll-like receptor 4 (TLR4) is a transmembrane protein that is a member of the toll-like receptor family, which belongs to 


\section{Cellular Physiology Cell Physiol Biochem 2018;45:2257-2267

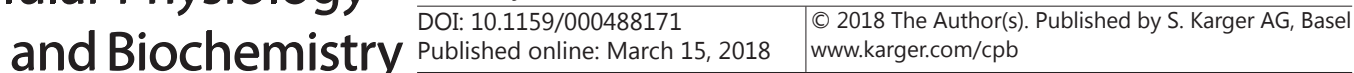 \\ Zhang et al.: Kudingcha Nanoparticles for the Treatment of Hyperlipidaemia}

the pattern recognition receptor (PRR) family. Its activation triggers the NF- $\kappa B$ intracellular signalling pathway and inflammatory cytokine production, which is responsible for activating the innate immune system [13]. Recently, TLR4 has also been shown to play an essential role in the pathogenesis of atherosclerosis-related insulin resistance [14], diet-induced obesity and hyperlipidaemia [15]. It has also been demonstrated that TLR4 deletion protects mice from NF- $\kappa B$ mediated inflammation [16]. Hence, the present study also evaluated whether the effect of Kudingcha nanoparticles on hyperlipidaemia is regulated by the TLR4-NFKB signalling pathway.

Therefore, in this study, we prepared Kudingcha nanoparticles and investigated the hypolipidaemic effect on rats fed high fat diets. Furthermore, the acute toxicity of the nanoparticles was examined, and the mechanism of Kudingcha on lipid metabolism was also evaluated.

\section{Materials and Methods}

\section{Materials}

Kudingcha was provided by Huang's Kudingcha Company (Daxin County, Guangxi Zhuang Autonomous Region, China). It was identified as one of the species in the Ilex genus by Wang Jie (senior technician of Guangxi Medical University). Chitosan with an average molecular weight of $210 \mathrm{kDa}$ was purchased from Shandong Aokang Biotech Ltd. (Shandong, China). The viscosity of the chitosan was less than $200 \mathrm{cP}$, and the deacetylation value was $96.2 \%$. TC, TG, HDL-C and LDL-C kits were obtained from Wako Pure Chemical Industries, Ltd. (Japan). Superoxide dismutase (SOD) and methane dicarboxylic aldehyde (MDA) kits were purchased from Nanjing Jiancheng Bioengineering Institute (Wenzhou, China). All other reagents and solvents were of analytical grade.

\section{Preparation and characterization of nanoparticles}

Kudingcha nanoparticles were prepared using previously reported methods [17]. In summary, chitosan was dissolved in $1 \%$ acetic acid, forming a solution at the concentration of $2.0 \mathrm{mg} / \mathrm{mL}$, and tripolyphosphate (TPP) was dissolved in distilled water at $1.0 \mathrm{mg} / \mathrm{mL}$. A specific volume of chitosan solution was obtained and slowly added into the total flavonoids of the Kudingcha solution at room temperature with magnetic stirring $(600 \mathrm{rpm})$. This mixed solution was slowly placed into the TPP solution using a 4th syringe needle for 45 min. Finally, a nanosuspension was spontaneously obtained when blue opalescence appeared [18]. Spray-drying was performed for the nanosuspension with a Lab Spray Dryer L-117 (Laiheng Scientific Co. Ltd, Beijing, China). The nozzle was $0.5 \mathrm{~mm}$, and the other adjustable parameters, including the inlet and outlet temperatures, solution pump flow rate, and aspirator partial vacuum, were similar to those in our previous studies $[19,20]$.

The particle size and size distributions of the nanoparticles were determined with a particle sizer (Zetasizer 3000 HAS, Malvern Instruments Ltd., Worcs, UK). The morphology of the nanoparticles was examined using transmission electron microscopy (H-7650, Japan Hitachi).

\section{Experimental protocols}

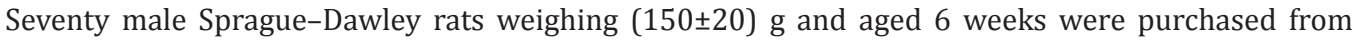
the Laboratory Animal Center of Guangxi Medical University (Nanning, China). During the experimental period, the animals were housed at the ratio of 8 rats per cage in environmentally controlled conditions (temperature $22^{\circ} \mathrm{C}, 12$-hour light-dark cycle) and allowed access to food and water ad libitum. All rats were fed standard rodent chow (provided by the Laboratory Animal Center of Guangxi Medical University, Nanning, China) and allowed to acclimatize for 1 week. Then, the rats were divided into the normal fat diet group (NF group, $n=8$ ) and high fat group $(n=62)$ to establish the hyperlipidaemia model. The NF group was fed standard rodent chow ad libitum, and the other group received the high-fat diet, which is explained in our previous report [20]. After 2 weeks, when the hyperlipidaemia model was successful, the 62 rats were randomly divided into seven groups of eight rats:

high fat group (HF);

spontaneous recovery group (SR); 


\section{Cellular Physiology Cell Physiol Biochem 2018;45:2257-2267 \begin{tabular}{c|c|c|}
\cline { 2 - 3 } DOI: 10.1159/000488171 & $\begin{array}{l}\text { O } 2018 \text { The Author(s). Published by S. Karger AG, Basel } \\
\text { www.karger.com/cpb }\end{array}$
\end{tabular} Zhang et al.: Kudingcha Nanoparticles for the Treatment of Hyperlipidaemia}

Kudingcha group (KDC);

blank nanoparticle group (B-N);

Kudingcha nanoparticle low-dose group (KDC-N-L);

Kudingcha nanoparticle medium-dose group (KDC-N-M);

Kudingcha nanoparticle high-dose group (KDC-N-H).

The NF group remained as the control group and was provided standard rodent chow ad libitum, and the HF group continued the high-fat diet until the study end. As the spontaneous recovery control, the SR group was only offered standard rodent chow. Aside from the SR group, all the other groups received a normal diet combined with the different interventions. Rats from the KDC and B-N groups received flavonoids of Kudingcha and blank nanoparticles, respectively, at the dose of $200 \mathrm{mg} / \mathrm{kg}$. Kudingcha nanoparticles were administered to the KDC-N-L, KDC-N-M and KDC-N-H groups at doses of 50, 100 and $200 \mathrm{mg} / \mathrm{kg}$, respectively. All the regimens were administered orally once daily for 4 weeks.

All animal protocols were approved by the institutional animal care and use committee of the First Affiliated Hospital of Guangxi Medical University (Nanning, China).

\section{Primary Outcome Measurements}

The rats body weights were recorded daily. At the end of the experimental period, the rats were fasted overnight, and blood samples were taken from the orbital vein using a capillary tube under ether anaesthesia to quantify the levels of antioxidants and lipids. Antioxidants, including SOD and MDA, were measured by the colourimetric method using a TU-1901 UV-Vis Spectrophotometer (Beijing Purkinje General Instrument Co., LTD). Serum lipids were assayed by the enzyme method using commercially available kits (Wako Pure Chemical Industries, Ltd) with an Automated Biochemistry Analyzer 7100 (Hitachi, Ltd).

Then, the rats were sacrificed and necropsied. Liver, epididymal and perirenal white adipose tissue were removed and weighed quickly. Liver morphology and histopathology were examined under a light microscope (Olympus, Tokyo, Japan). Tissue sections were stained with haematoxylin and eosin and observed at $20 \times$ magnification.

\section{Relative quantitative real-time PCR}

The total RNA of liver tissue was extracted using the RNAiso Plus kit (Takara Biotechnology Co. Ltd, Dalian, China) according to the manufacturer's protocols. cDNA was synthesized from RNA with a PrimeScript $^{\mathrm{TM}}$ RT reagent Kit (Takara Biotechnology Co. Ltd, Dalian, China). PCR was conducted on an ABI Prism 7300 real-time thermocycler (Applied Biosystems, Foster City, CA, USA) using the TransScript ${ }^{\mathrm{TM}}$ SYBR ${ }^{\circledR}$ Green Master Mix kit (Takara Biotechnology Co. Ltd, Dalian, China). The different pairs of oligonucleotide primers used for PCR are shown in Table 1. $\beta$-actin was used as an endogenous control. The amount of gene expression was normalized by the $2^{-\Delta \Delta \mathrm{CT}}$ relative quantitative method.

\section{Western blotting analysis}

Liver tissues were lysed with PMSF and RIPA solutions. Protein concentration was determined using the BCA protein quantification kit (Bio-Rad, USA). Western blot was performed as previously described [21]. Proteins were detected with primary antibodies to TLR4 and NF- $\kappa B$ (Cell Signaling Technology, Boston, MA, USA). The membranes were scanned under an infrared imaging system (Odyssey, LI-COR, USA).

\section{Acute oral toxicity}

Acute oral toxicities of the Kudingcha nanoparticles were evaluated in mice using the methods that we previous reported [20]. Briefly, the mice were divided into the control group and 4 treatment groups, with 10 animals in each group (half male and half female). The control groups received normal saline, and the treatment groups were administered 1000, 2150, 4640, and 10, $000 \mathrm{mg} / \mathrm{kg}$ of the Kudingcha nanoparticles. The mortality and symptoms were observed and measured for 14 days after oral administration of the different dosages. Finally, the rats were sacrificed by cervical dislocation. Vital organs, including the liver, lung, spleen, kidneys, and heart, were removed for macroscopic analysis.

Table 1. Primers information for RT-PCR

\begin{tabular}{lccc}
\hline Gene & Forward primer $\left(5^{\prime}-3^{\prime}\right)$ & Reverse primer $\left(5^{\prime}-3^{\prime}\right)$ & Product size (bp) \\
\hline TLR4 & CTCCTGCGTGAGACCAGAAA & AGGAACCACCTCCGTGATAAA & 169 \\
NFkB & AGCTTGTAGGAAAGGACTGCC & AGGGGTTGTTGTTGGTCTGG & 156 \\
\multirow{2}{*}{-actin } & GGCATCCTCACCCTGAAGTA & GCACACGCAGCTCATTGTAG & 103 \\
\hline
\end{tabular}




\section{Cellular Physiology Cell Physiol Biochem 2018;45:2257-2267 \begin{tabular}{l|l} 
DOI: 10.1159/000488171 & and Biochemistry \\
Published online: Narch 15, 2018 & $\begin{array}{l}\text { O } 2018 \text { The Author(s). Published by S. Karger AG, Basel } \\
\text { www.karger.com/cpb }\end{array}$
\end{tabular} \\ Zhang et al.: Kudingcha Nanoparticles for the Treatment of Hyperlipidaemia}

Statistical analysis

All statistical analyses were performed using SPSS software version 19.0 (SPSS, Inc., Chicago, IL, USA), and the data are expressed as the mean and standard deviation (SD). One-way analysis of variance (ANOVA) with the Student-Newman-Keuls multiple comparison test was used to examine differences between two groups. A value of $p<0.05$ was considered statistically significant.

\section{Results}

Characterization of the Kudingcha nanoparticles

The shape of the Kudingcha nanoparticles was spherical, and the surface was smooth (Fig. 1). The particle size distribution of the nanoparticles was between 100 and $600 \mathrm{~nm}$, and the distribution was concentrated with a dispersion coefficient of 0.137 . All these characters have been described in our previous stud [17].

\section{Serum lipids}

In comparison with the NF group, the serum levels of TG, TC and LDL-C increased significantly $(p<0.05)$ in the high-fat diet group after two weeks, which implied that the hyperlipidaemia model was successful. The results for serum TC, TG, HDL-C, and LDL-C after four weeks of

Table 2. Effects of KDC-N on serum lipids in rats fed a high-fat diet (mean \pm SD $n=8$ ), Notes: \# $\mathrm{p}<0.05$, versus the NF group; $\star \mathrm{p}<0.05$, versus the HF group; ${ }^{*}<<0.05$, versus the $S R$ group; $\Delta p<0.05$, versus the KDC group; $+\mathrm{p}<0.05$, versus the B-N group; Abbreviations: $\mathrm{NF}$, normal fat diet group; HF, high fat diet group; SR, spontaneous recovery group; KDC, Kudingcha group; B-N, blank nanoparticles group; KDC-N-L, Kudingcha nanoparticles low-dose group; KDC-N-M, Kudingcha nanoparticles medial-dose group; KDC-N-H, Kudingcha nanoparticles high dose group

\begin{tabular}{|c|c|c|c|c|}
\hline Groups & $\mathrm{TC}(\mathrm{mmol} / \mathrm{L})$ & TG $(\mathrm{mmol} / \mathrm{L})$ & HDL-C $(\mathrm{mmol} / \mathrm{L})$ & LDL-C $(\mathrm{mmol} / \mathrm{L})$ \\
\hline NF & $1.92 \pm 0.22^{*}$ & $2.22 \pm 0.56^{* *}$ & $1.68 \pm 0.19$ & $0.41 \pm 0.13$ \\
\hline $\mathrm{HF}$ & $3.04 \pm 0.37$ & $3.33 \pm 0.70$ & $1.73 \pm 0.14$ & $0.67 \pm 0.25$ \\
\hline SR & $2.23 \pm 0.45^{*}$ & $3.27 \pm 1.04$ & $1.59 \pm 0.27$ & $0.46 \pm 0.29$ \\
\hline $\mathrm{KDC}$ & $2.33 \pm 0.47^{*}$ & $1.99 \pm 0.60^{* *}$ & $1.93 \pm 0.32$ & $0.75 \pm 0.32$ \\
\hline B-N & $2.36 \pm 0.43^{*}$ & $2.18 \pm 0.31^{* *}$ & $1.84 \pm 0.26$ & $0.66 \pm 0.22$ \\
\hline KDC-N-L & $2.23 \pm 0.38^{*}$ & $2.05 \pm 0.41^{* *}$ & $1.80 \pm 0.30$ & $0.60 \pm 0.23$ \\
\hline KDC-N-M & $2.10 \pm 0.14^{*}$ & $2.09 \pm 0.50$ ** & $1.77 \pm 0.16$ & $0.65 \pm 0.21$ \\
\hline KDC-N-H & $1.94 \pm 0.19^{*_{*} \star+}$ & $1.56 \pm 0.23^{*^{*}} \Delta$ & $1.65 \pm 0.19$ & $0.72 \pm 0.27$ \\
\hline
\end{tabular}
treatment are shown in Table

2. Compared with the HF group, TC and TG were significantly reduced in the Kudingcha nanoparticle groups $(p<0.05)$. The effect was more pronounced when the dosage of nanoparticles increased. In the KDC$\mathrm{N}-\mathrm{H}$ group, TG and TC were also distinctly decreased in comparison with the SR, KDC, and B-N groups. Noticeably, TC was reduced in the SR group compared with the HF group; however, the same response was not observed for TG. The results indicated that TC had the ability for spontaneous recovery in hyperlipidaemic rats, but TG did not exhibit this ability. In addition, Kudingcha nanoparticles did not play a great role in the HDL-C and LDL-C levels.

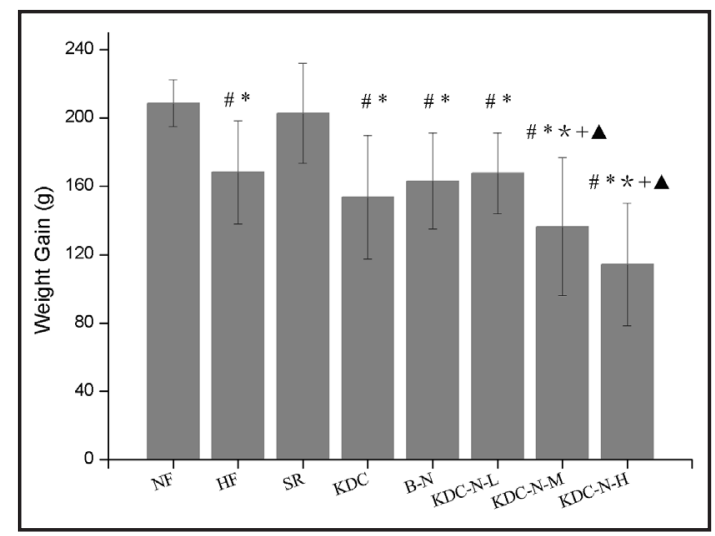

Fig. 2. Kudingcha nanoparticles lower body weight in hyperlipidaemic rats( mean $\pm \mathrm{SD}, \mathrm{n}=8$ ). Note: \# $\mathrm{p}<0.05$, versus the NF group; $* \mathrm{p}<0.05$, versus the HF group; ${ }^{*} \mathrm{p}<0.05$, versus the SR group; $\Delta \mathrm{p}<0.05$, versus the KDC group; $+\mathrm{p}<0.05$, versus the $\mathrm{B}-\mathrm{N}$ group. 


\section{Cellular Physiology Cell Physiol Biochem 2018;45:2257-2267 \begin{tabular}{l|l} 
DOI: 10.1159/000488171 & and Biochemistry \\
Published onl'ne: Narch 15, 2018 & $\begin{array}{l}\text { O } 2018 \text { The Author(s). Published by S. Karger AG, Basel } \\
\text { www.karger.com/cpb }\end{array}$
\end{tabular} \\ Zhang et al.: Kudingcha Nanoparticles for the Treatment of Hyperlipidaemia}

Body, liver and adipose tissue weights

The changes of body weight in different groups after 4 weeks of treatment are shown in Fig. 2. In contrast the SR group, the KDC-N-L, KDC-N-M, KDC-N-H, KDC, and $\mathrm{B}-\mathrm{N}$ groups showed a lower body weight compared with the NF group $(p<0.05)$. Furthermore, compared with the KDC and B-N groups, the KDC-N-M and KDC$\mathrm{N}-\mathrm{H}$ groups also displayed a better effect in terms of weight loss $(p<0.05)$, which was dose related. No body weight gain was observed in the NF and SR groups $(p>0.05)$. Intriguingly, a significantly lower weight gain was observed in the HF group compared with the NF and SR groups. This situation might be attributed to the lack of appetite, which may be caused by a long-term high fat diet.

The final liver and adipose tissue weights of the groups are shown in Table 3. The liver weight was obviously reduced in the five treatment groups (KDC, B-N, KDC$\mathrm{N}-\mathrm{L}, \mathrm{KDC}-\mathrm{N}-\mathrm{M}$ and KDC$\mathrm{N}-\mathrm{H}$ ) compared to the $\mathrm{NF}, \mathrm{HF}$ and SR groups $(p<0.05)$. Analogously, adipose tissue weights, including epididymal and perirenal white adipose tissue, was decreased in the five treatment groups, and moderate and high doses of Kudingcha nanoparticles were more effective than the other treatment doses.

\section{Antioxidation}

Serum SOD was significantly increased in all the treatment groups compared with the HF and SR groups $(p<0.05)$, and a distinct difference was also revealed between the KDC-N-H group and the KDC group (Fig. 3a). Liver SOD levels were higher

Fig. 4. Effect of Kudingcha nanoparticles on liver morphologies in the hyperlipidaemic rats.
Table 3. Liver weights and adipose tissues in rats $($ mean $\pm S D, n=8$ ). Note: \# $<<0.05$, versus the $N F$ group; $\star \mathrm{p}<0.05$, versus the HF group; ${ }^{*} \mathrm{p}<0.05$, versus the SR group; $\Delta \mathrm{p}<0.05$, versus the KDC group; + $\mathrm{p}<0.05$, versus the $\mathrm{B}-\mathrm{N}$ group

\begin{tabular}{lcc}
\hline Groups & liver weights $(\mathrm{g})$ & adipose tissues weight $(\mathrm{g})$ \\
\hline $\mathrm{NF}$ & $13.83 \pm 1.48$ & $7.22 \pm 1.16$ \\
$\mathrm{HF}$ & $13.66 \pm 1.86$ & $9.71 \pm 3.07$ \\
$\mathrm{SR}$ & $13.57 \pm 0.87$ & $7.41 \pm 3.34$ \\
KDC & $11.20 \pm 1.49^{\#^{* *}}$ & $5.64 \pm 1.77^{*}$ \\
B-N & $11.99 \pm 1.18^{\#^{* *}}$ & $5.39 \pm 1.00^{*}$ \\
KDC-N-L & $11.76 \pm 1.22^{\#^{* *}}$ & $6.80 \pm 1.86^{*}$ \\
KDC-N-M & $10.58 \pm 1.88^{\#^{* *}}$ & $4.04 \pm 2.00^{\#^{* *}}$ \\
KDC-N-H & $10.05 \pm 1.20^{\#^{* *}}$ & $3.29 \pm 0.94^{\#^{* *}}$ \\
\hline
\end{tabular}

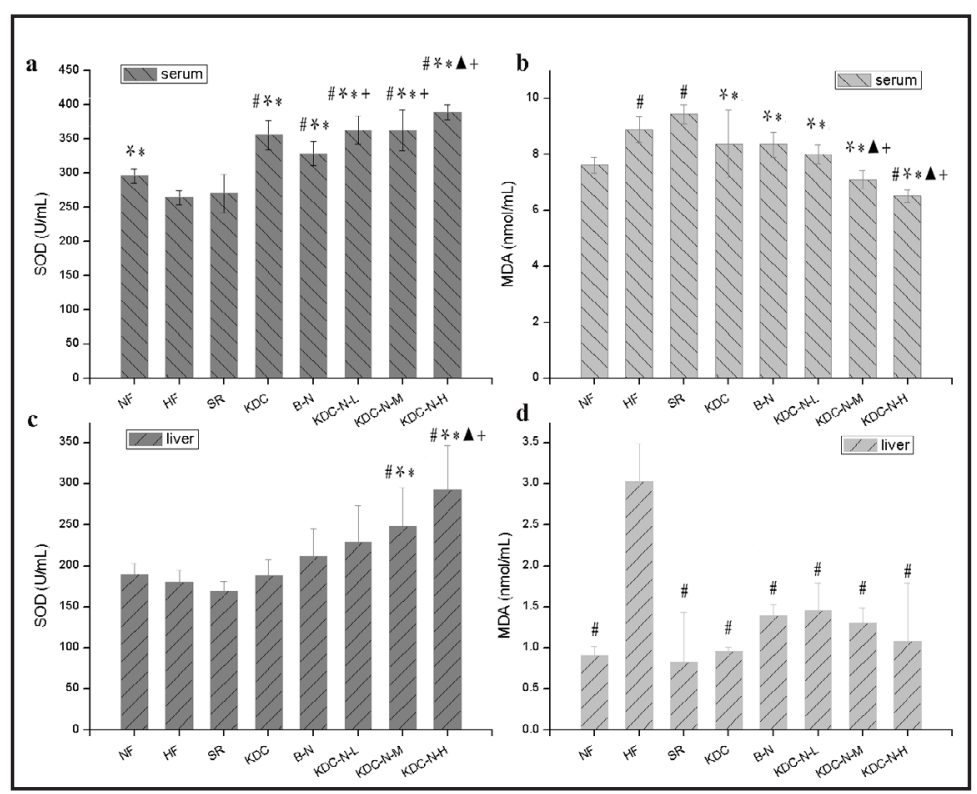

Fig. 3. Kudingcha nanoparticles impact the content of SOD and MDA in the serum and liver of hyperlipidaemic rats (mean $\pm S D, n=8$ ). (a) The SOD in serum; (b) the MDA in serum; (c) the SOD in the liver; (b) the MDA in the liver. Note: $\# \mathrm{p}<0.05$, versus the NF group; $* \mathrm{p}<0.05$, versus the HF group; ${ }^{*} \mathrm{p}<0.05$, versus the SR group; $\mathbf{\Delta} \mathrm{p}<0.05$, versus the KDC group; + $\mathrm{p}<0.05$, versus the $\mathrm{B}-\mathrm{N}$ group.

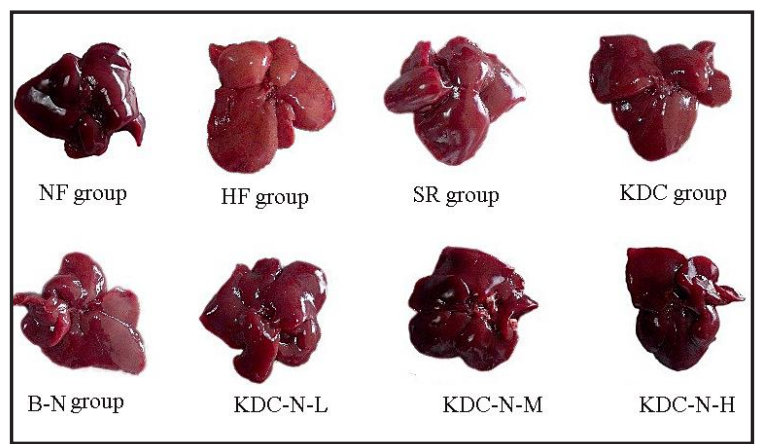




\section{Cellular Physiology Cell Physiol Biochem 2018;45:2257-2267 \begin{tabular}{ll|l} 
DOI: 10.1159/000488171 & Ond Biochemistry & 2018 The Author(s). Published by S. Karger AG, Basel \\
www.karger.com/cpb
\end{tabular} \\ Zhang et al.: Kudingcha Nanoparticles for the Treatment of Hyperlipidaemia}

for the middle and high dose nanoparticle groups compared with the HF, NF, and SR groups $(p<0.05)$. No difference was observed in the other groups (Fig. 3c). In addition, the serum malondialdehyde (MDA) value for all treatment groups was obviously decreased compared to the HF and SR groups $(p<0.05)$. The blood MDA level in the KDC-N-M and KDC-N-H groups was particularly lower than that of the controls $(p<0.001)$. The comparative results are provided in Fig. 3b. However, the situation was different in the liver. Compared with the HF group, all the other groups manifested a significant decrease in the MDA level. There were no differences revealed among the other groups (Fig. 3d).

\section{Liver morphology and histology}

The results for liver morphology are shown in Fig. 4. Consistent with the NF group, the livers of rats in the KDC-N-M and KDC-N-H groups were relatively small, crimson in colour, and featured sharp edges and smooth sections. By comparison, those in the HF group were light red, swollen, and had thick edges and apparent fat granules. The results indicated that KDC nanoparticles at moderate and high doses have a protective effect on the liver through reductions in fat deposition and acceleration of fat metabolism in the liver in rats.

The liver histology (Fig. 5) showed that the livers of rats in the KDC-N-L, KDC-N-M and KDC-N-H groups contained few fat droplets and necrotic cells, similar to those in the NF group. In contrast, the rats in the HF group developed severe steatosis; the histology of the liver exhibited a large number of fat vacuoles, and these hepatocytes fused together, implying that the hyperlipidaemia model was successfully established. Both the KDC and B-N groups revealed slightly alleviated signs of fatty liver compared to the Kudingcha nanoparticle groups. These results demonstrated that Kudingcha nanoparticles have a positive effects on the fatty liver in rats.

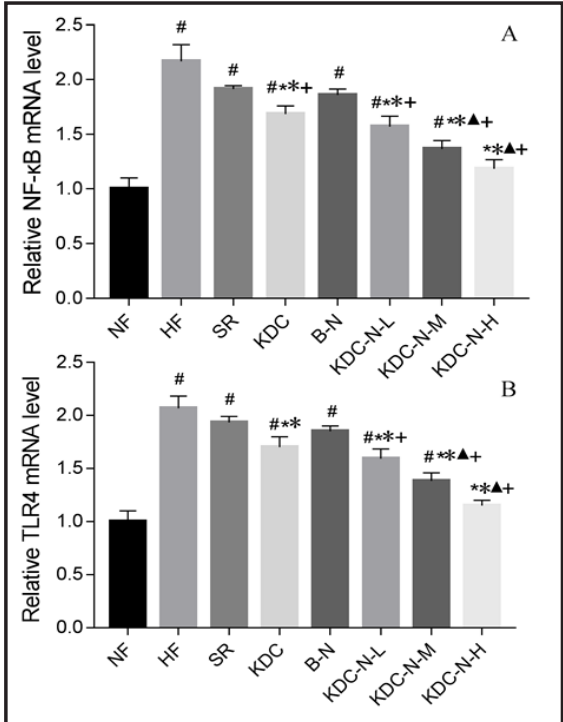

Fig. 6. Kudingcha nanoparticles downregulate the mRNA expression of TLR4 and NF- $\kappa B$ in liver tissue. (A) mRNA expression of NF- $\kappa$ B in liver tissue; (B) mRNA expression of TLR4 in liver tissue. Note: $\# \mathrm{p}<0.05$, versus the NF group; $* \mathrm{p}<0.05$, versus the HF group; ${ }^{*} \mathrm{p}<0.05$, versus the SR group; $\Delta \mathrm{p}<0.05$, versus the KDC group; $+\mathrm{p}<0.05$, versus the $\mathrm{B}-\mathrm{N}$ group.

\section{TLR4 and NF- $\kappa B$ mRNA expression levels in liver tissue}

PCRanalysisshowed that the mRNA expressionlevels ofTLR 4 andNF- $\kappa$ B were significantly upregulated in the liver tissue of the groups induced by a high fat diet compared with the normal fat diet group $(p<0.05)$. Following the Kudingcha nanoparticle treatment, the mRNA expression levels of TLR4 and NF- $\kappa B$ were reduced, particularly in the rats administered the high dose of KDC nanoparticles $(p<0.05)$. Compared with the KDC group, the medium and high 
doses of nanoparticles revealed more obvious downregulation in the mRNA expression levels of TLR4 and NF- $\kappa B \quad(p<0.05)$. In addition, the mRNA levels in the spontaneous recovery and blank nanoparticle groups did not exhibit an evident decrease compared to the high fat diet group ( $p>0.05)$. These results are presented in Fig. 6.

TLR4 and NF- $\kappa B$ protein expression levels in liver tissue

The protein expression levels of TLR4 and NF- $\kappa B$ are shown in Fig. 7. Compared with the normal control group, the protein levels of TLR4 and NF-

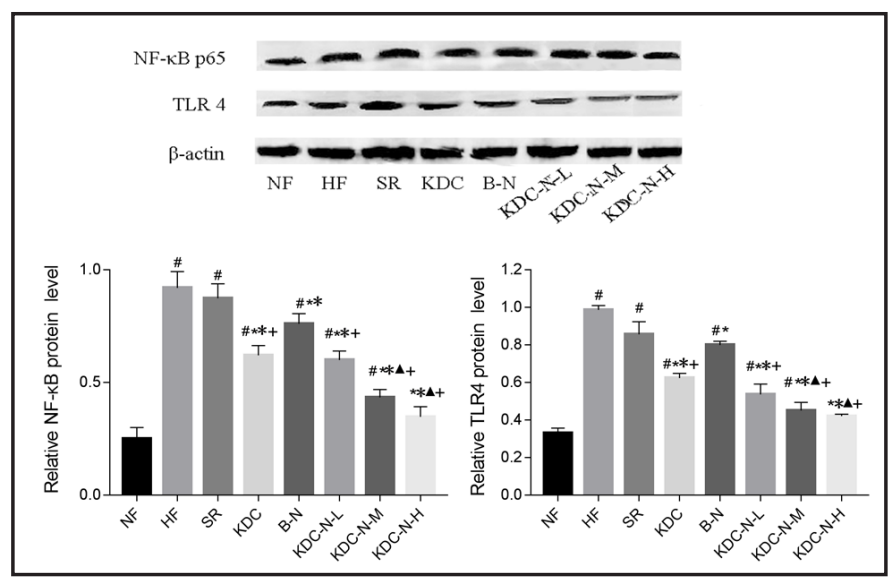

Fig. 7. Kudingcha nanoparticles downregulate the protein expression of TLR4 and NF- $\kappa B$ in liver tissue. Note: $\# p<0.05$, versus the $\mathrm{NF}$ group; $* \mathrm{p}<0.05$, versus the HF group; ${ }^{*} \mathrm{p}<0.05$, versus the SR group; $\Delta \mathrm{p}<0.05$, versus the KDC group; $+\mathrm{p}<0.05$, versus the $\mathrm{B}-\mathrm{N}$ group. $\kappa \mathrm{B}$ were obviously upregulated in the high fat diet groups $(p<0.05)$. After treatment with the KDC nanoparticles, the protein levels were decreased, particularly in the rats administered the high dose of KDC nanoparticles $(p<0.05)$. Similarly, medium and high doses of nanoparticles displayed more apparent downregulation than the regular KDC in the protein expression levels of TLR4 and $\mathrm{NF}-\kappa \mathrm{B}(p<0.05)$. Likewise, the protein levels in the spontaneous recovery group were not decreased compared to the high fat diet group $(p>0.05)$.

\section{Acute toxicity}

The number of deaths for all doses tested was less than half. Therefore, the median lethal dose $\left(\mathrm{LD}_{50}\right)$ of $\mathrm{KDC}$ nanoparticles was over $10,000 \mathrm{mg} / \mathrm{kg}$. This implies that KDC nanoparticles did not cause any acute toxicity.

\section{Discussion}

The polymers utilized in nanoparticles that are currently in clinical use are generally accepted as being biocompatible and safe. In this study, chitosan was used as a polymer carrier to prepare the nanoparticles. As a well-known natural polyelectrolyte, chitosan has attracted interest as a biocompatible, biodegradable, mucoadhesive, and nontoxic material for use in biomedical applications [22]. However, formulations of different nanoparticle sizes might have different physicochemical properties; thus, toxicities must be considered. The aim of an acute oral toxicity study is the estimation of $\mathrm{LD}_{50}$, which is currently the basis for the toxicological classification of chemicals. The $\mathrm{LD}_{50}$ of Kudingcha nanoparticles was over $10,000 \mathrm{mg} / \mathrm{kg}$, a value that is considered as safe [20].

Hyperlipidaemia is a major risk factor for cardiovascular disease, and statin-based lowering of LDL cholesterol has demonstrated a significant reduction in cardiovascular disease events in patients with hyperlipidaemia [23]. However, some patients who have achieved low LDL cholesterol levels remain at high residual cardiovascular disease risk, especially if TG levels are elevated [24]. This may be due to other risk factors beyond LDL cholesterol resulting in the situation. Over the past few decades, age-adjusted LDL-C has fallen significantly following the increased use of statins, and a significant increase in TG levels has become increasingly common throughout the world [25]. Furthermore, research has shown that obese and overweight individuals have higher TG levels than the normal- 


\section{Cellular Physiology Cell Physiol Biochem 2018;45:2257-2267

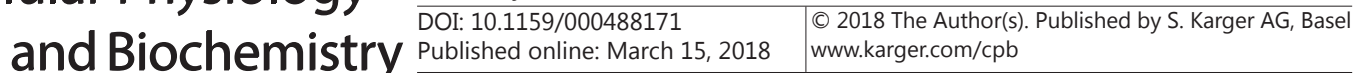 \\ Zhang et al.: Kudingcha Nanoparticles for the Treatment of Hyperlipidaemia}

weight population [26]. In addition, hypertriglyceridaemia is also common in patients with the metabolic syndrome, type 2 diabetes, and renal disease [27].

In the past few years, some studies have linked high TG levels to an increased risk of cardiovascular disease events, including ischaemic stroke, myocardial infarction, ischaemic heart disease, and death in adults [28]. In this study, Kudingcha nanoparticles did not exhibit a notable role in reducing LDL-C. However, Kudingcha was found to exhibit outstanding capacities for lowering TC and TG in hyperlipidaemic rats. Simultaneously, the TC level was also reduced in the spontaneous recovery group compared with the high fat diet group. This indicates that Kudingcha nanoparticles only exhibited the ability to decrease TG and implies that they would be a promising agent for the treatment of hypertriglyceridaemia.

In hyperlipidaemic patients, high cholesterol not only is correlated with adipocyte cholesterol but also has been shown to regulate adipocyte development [29]. Accordingly, patients with hyperlipidaemia are often obese. Our research revealed that KDC-NPs visibly lowered body weight, adipose tissue and liver weight compared with regular KDC.

As a marker for oxidative stress, MDA results from lipid peroxidation of polyunsaturated fatty acids [30]. The degree of lipid peroxidation can be estimated by the amount of MDA in tissue. SOD is an enzyme that catalyses the dismutation of the superoxide radical into either ordinary molecular oxygen or hydrogen peroxide [31]. Superoxide is produced as a by-product of oxygen metabolism and, if not regulated, causes many types of cell damage. Our studies revealed that liver and serum SOD levels increased significantly in the Kudingcha nanoparticle groups. Conversely, serum MDA values were decreased in the nanoparticle groups. In light of this, Kudingcha nanoparticles may be a new therapy for the treatment of diseases involving oxidative stress.

Liver morphology and histology showed that KDC nanoparticles at moderate and high doses have a protective effect on the fatty liver in rats. Moreover, the mRNA and protein expression levels of TLR4 and NF- $\kappa B$ were significantly downregulated in the liver tissue by the KDC nanoparticle treatments, particularly in the rats administered a high dose of nanoparticles. These outcomes indicate that the mechanism of KDC nanoparticles for the treatment of hyperlipidaemia, especially for fatty liver, may occur via the TLR4-NFKB signalling pathway. It is of great necessity to conduct RNAi and overexpression analysis in future research to elucidate the role of the TLR4-NFKB signalling pathway in the use of KDC nanoparticles for the treatment of hyperlipidaemia.

\section{Acknowledgements}

This research was supported by Guangxi Natural Science Foundation under Grant No. 2017GXNSFBA198177. In addition, this project was financially supported by the Guangxi Zhuang Autonomous Region Health Department of Traditional Chinese Medicine Science and Technology projects, Guangxi Province, China (No. GZZJ13-17 and S2017016); the project of improving the basic ability of young teachers in colleges and universities in Guangxi (KY2016YB098); and the Innovation Project of Guangxi Graduate Education (YCBZ2017043).

\section{Disclosure Statement}

The authors declare no conflicts of interest in this work.

\section{References}

\footnotetext{
1 Skoumas I, Masoura C, Aznaouridis K, Metaxa V, Tsokanis A, Papadimitriou L, Tousoulis D, Pitsavos C, Stefanadis C: Impact of cardiometabolic risk factors on major cardiovascular events in patients with familial combined hyperlipidemia. Circ J 2013;77:163-168.
} 


\section{Cellular Physiology Cell Physiol Biochem 2018;45:2257-2267

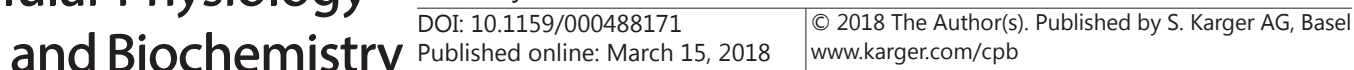

-2 Smith AG, Singleton JR: Obesity and hyperlipidemia are risk factors for early diabetic neuropathy. J Diabetes Complicat 2013;27:436-442.

3 Nelson RH: Hyperlipidemia as a risk factor for cardiovascular disease. Primary Care 2013;40:195-211.

4 Catapano AL: Statin-induced myotoxicity: pharmacokinetic differences among statins and the risk of rhabdomyolysis, with particular reference to pitavastatin. Curr Vasc Pharmacol 2012;10:257-267.

5 Lee CY, Yu MC, Lin CC, Lee MY, Wei JC, Shih HC: Efficacy and safety of herbal medicine yun-cai tea in the treatment of hyperlipidemia: A double-blind placebo-controlled clinical trial. Chin J Integr Med 2015;21:587-593.

-6 Bahmani M, Mirhoseini M, Shirzad H, Sedighi M, Shahinfard N, Rafieian-Kopaei M: A review on promising natural agents effective on hyperlipidemia. J Evid Based Complementary Altern Med 2015;20:228-238.

7 Liu ZL, Li GQ Bensoussan A, Kiat H, Chan K, Liu JP: Chinese herbal medicines for hypertriglyceridaemia. Cochrane Db Syst Rev 2013:CD009560.

8 Li L, Xu LJ, Ma GZ, Dong YM, Peng Y, Xiao PG: The large-leaved Kudingcha (Ilex latifolia Thunb and Ilex kudingcha C.J. Tseng): a traditional Chinese tea with plentiful secondary metabolites and potential biological activities. J Nat Med 2013;67:425-437.

Lee MS, Dees EC, Wang AZ: Nanoparticle-Delivered Chemotherapy: Old Drugs in New Packages. Oncology (Williston Park) 2017;31:198-208.

10 Ohara Y, Oda T, Yamada K, Hashimoto S, Akashi Y, Miyamoto R, Kobayashi A, Fukunaga K, Sasaki R, Ohkohchi N: Effective delivery of chemotherapeutic nanoparticles by depleting host Kupffer cells. Int J Cancer 2012;131:2402-2410.

11 Jahangirian H, Lemraski EG, Webster TJ, Rafiee-Moghaddam R, Abdollahi Y: A review of drug delivery systems based on nanotechnology and green chemistry: green nanomedicine. Int J Nanomedicine 2017;12:2957-2978.

12 Nel A, Xia T, Madler L, Li N: Toxic potential of materials at the nanolevel. Science 2006;311:622-627.

-13 Janssens S, Beyaert R: Role of Toll-like receptors in pathogen recognition. Clin Microbiol Rev 2003;16:637646.

14 Bozzetto L, De Natale C, Di Capua L, Della Corte G, Patti L, Maione S, Riccardi G, Rivellese AA, Annuzzi G: The association of hs-CRP with fasting and postprandial plasma lipids in patients with type 2 diabetes is disrupted by dietary monounsaturated fatty acids. Acta Diabetol 2013;50:273-276.

15 Chen S, Lin G, Lei L, You X, Wu C, Xu W, Huang M, Luo L, Wang Z, Li Y, Zhao X, Yan F: Hyperlipidemia modifies innate immune responses to lipopolysaccharide via the TLR-NF-kappaB signaling pathway. Inflammation 2013;36:968-976.

16 Shi H, Kokoeva MV, Inouye K, Tzameli I, Yin H, Flier JS: TLR4 links innate immunity and fatty acid-induced insulin resistance. J Clin Invest 2006;116:3015-3025.

17 Zhang H, Huang Q Huang Z, Liu T, Li Y: Preparation and physicochemical properties of chitosan broadleaf holly leaf nanoparticles. Int J Pharm 2015;479:212-218.

18 Elsayed A, Al-Remawi M, Qinna N, Farouk A, Al-Sou'od KA, Badwan AA: Chitosan-sodium lauryl sulfate nanoparticles as a carrier system for the in vivo delivery of oral insulin. AAPS PharmSciTech 2011;12:958964.

19 Zhang HL, Tao Y, Guo J, Hu YM, Su ZQ: Hypolipidemic effects of chitosan nanoparticles in hyperlipidemia rats induced by high fat diet. Int Immunopharmacol 2011;11:457-461.

-20 Zhang HL, Zhong XB, Tao Y, Wu SH, Su ZQ: Effects of chitosan and water-soluble chitosan micro- and nanoparticles in obese rats fed a high-fat diet. Int J Nanomedicine 2012;7:4069-4076.

21 Zhou X, Sun X, Gong X, Yang Y, Chen C, Shan G, Yao Q: Astragaloside IV from Astragalus membranaceus ameliorates renal interstitial fibrosis by inhibiting inflammation via TLR4/NF-small ka, CyrillicB in vivo and in vitro. Int Immunopharmacol 2017;42:18-24.

22 Zhang HL, Wu SH, Tao Y, Zang LQ, Su ZQ: Preparation and Characterization of Water-Soluble Chitosan Nanoparticles as Protein Delivery System. J Nanomater 2010; DOI:10.1155/2010/898910.

23 Grutzmacher P, Ohm B, Szymczak S, Dorbath C, Brzoska M, Kleinert C: Primary and secondary prevention of cardiovascular disease in patients with hyperlipoproteinemia (a). Clin Res Cardiol Suppl 2017;12:22-26. 


\section{Cellular Physiology Cell Physiol Biochem 2018;45:2257-2267 \begin{tabular}{l|l} 
DOI: 10.1159/000488171 & $\begin{array}{l}\text { O 2018 The Author(s). Published by S. Karger AG, Basel } \\
\text { www.karger.com/cpb }\end{array}$ \\
\hline
\end{tabular} \\ Zhang et al.: Kudingcha Nanoparticles for the Treatment of Hyperlipidaemia}

-24 Faergeman O, Holme I, Fayyad R, Bhatia S, Grundy SM, Kastelein JJ, LaRosa JC, Larsen ML, Lindahl C, Olsson AG, Tikkanen MJ, Waters DD, Pedersen TR, Steering Committees of I, Trials TNT: Plasma triglycerides and cardiovascular events in the Treating to New Targets and Incremental Decrease in End-Points through Aggressive Lipid Lowering trials of statins in patients with coronary artery disease. Am J Cardiol 2009;104:459-463.

25 Karalis DG: A Review of Clinical Practice Guidelines for the Management of Hypertriglyceridemia: A Focus on High Dose Omega-3 Fatty Acids. Adv Ther 2017;34:300-323.

-26 Carroll M, Kit B, Lacher D: Trends in elevated triglyceride in adults: United States, 2001-2012 NCHS Data Brief 2015:198.

-27 Resnick HE, Foster GL, Bardsley J, Ratner RE: Achievement of American Diabetes Association clinical practice recommendations among U.S. adults with diabetes, 1999-2002: the National Health and Nutrition Examination Survey. Diabetes Care 2006;29:531-537.

28 Freiberg JJ, Tybjaerg-Hansen A, Jensen JS, Nordestgaard BG: Nonfasting triglycerides and risk of ischemic stroke in the general population. JAMA 2008;300:2142-2152.

29 Aguilar D, Fernandez ML: Hypercholesterolemia induces adipose dysfunction in conditions of obesity and nonobesity. Adv Nutr 2014;5:497-502.

-30 Davey MW, Stals E, Panis B, Keulemans J, Swennen RL: High-throughput determination of malondialdehyde in plant tissues. Anal Biochem 2005;347:201-207.

-31 Hayyan M, Hashim MA, AlNashef IM: Superoxide Ion: Generation and Chemical Implications. Chem Rev 2016;116:3029-3085. 